\title{
Cortical Acetylcholine Release Is Lateralized during Asymmetrical Slow-Wave Sleep in Northern Fur Seals
}

\author{
Jennifer L. Lapierre, ${ }^{1}$ Peter 0. Kosenko, ${ }^{2}$ Oleg I. Lyamin, ${ }^{1,2}$ Tohru Kodama, ${ }^{3}$ Lev M. Mukhametov, ${ }^{2}$ and \\ Jerome M. Siegel ${ }^{1}$ \\ ${ }^{1}$ Department of Psychiatry, School of Medicine, University of California Los Angeles and Neurobiology Research, Veterans Affairs Greater Los Angeles \\ Healthcare System, North Hills, California 91343, ${ }^{2}$ Utrish Dolphinarium Ltd., Moscow, Russia 119071, and ${ }^{3}$ Department of Psychology, Tokyo Metropolitan \\ Institute for Neuroscience, Tokyo, Japan 183-8526
}

Fur seals are unique in that they display both bilateral slow-wave sleep (BSWS), as seen in all terrestrial mammals, and slow-wave sleep with interhemispheric electroencephalogram (EEG) asymmetry, resembling the unihemispheric slow waves of cetaceans. Little is known about the underlying mechanisms of this phenomenon, which is also termed asymmetrical slow wave sleep (ASWS). However, we may begin to understand the expression of ASWS by studying the neurotransmitter systems thought to be involved in the generation and maintenance of sleep-wake states in terrestrial mammals. We examined bilaterally the release of cortical acetylcholine (ACh), a neurotransmitter implicated in the regulation of cortical EEG and behavioral arousal, across the sleep-wake cycle in four juvenile northern fur seals (Callorhinus ursinus). In vivo microdialysis and high-performance liquid chromatography coupled with electrochemical detection were used to measure cortical ACh levels during polygraphically defined behavioral states. Cortical ACh release was state-dependent, showing maximal release during active waking $(\mathrm{AW})$, similar levels during quiet waking $(\mathrm{QW})$, and rapid eye movement (REM) sleep, and minimal release during BSWS. When compared with BSWS, cortical ACh levels increased $\sim 300 \%$ during AW, and $\sim 200 \%$ during QW and REM sleep. During these bilaterally symmetrical EEG states, ACh was synchronously released from both hemispheres. However, during ASWS, ACh release was lateralized with greater release in the hemisphere displaying lower voltage activity, at levels approximating those seen in QW. These findings demonstrate that cortical ACh release is tightly linked to hemispheric EEG activation.

Key words: sleep-wake cycle; acetylcholine; microdialysis; cerebral cortex; asymmetry; Callorhinus ursinus

\section{Introduction}

All terrestrial mammals studied to date display bilaterally symmetrical synchronized electroencephalogram (EEG) activity during slow-wave sleep (SWS). Cetaceans (whales and dolphins), however, exhibit unihemispheric slow-wave sleep (USWS), in which slow-wave activity (SWA) is recorded in one hemisphere while a low-voltage EEG is recorded simultaneously in the other hemisphere (Mukhametov et al., 1977; Mukhametov 1984, 1987; Lyamin et al., 2002a; Ridgway, 2002). Unlike fully terrestrial or aquatic mammals, fur seals live and sleep both on land and in water. While sleeping on land, fur seals display predominantly bilateral slow-wave sleep (BSWS; 69\% of all SWS), as seen in terrestrial mammals (Lyamin and Mukhametov, 1998). However, when sleep occurs in water, fur seals primarily display SWS with interhemispheric EEG asymmetry (68\% of all SWS), which

Received June 29, 2007; revised Sept. 10, 2007; accepted Sept. 10, 2007.

This work was supported by Defense Advanced Research Projects Agency Grant BAA0412F8034, National Science Foundation Grant 0234687, National Institutes of Health Grant NS42947, the Medical Research Service of the Department of Veterans Affairs, and the Utrish Dolphinarium. We thank S. Azuma for HPLC technical support, J. Pryaslova for laboratory assistance, E. Rozanova and the personnel of the Utrish Marine Station for veterinary and animal care, and A. Vosko, K. Abordo, A. Blouin, and Z. Peterfi for invaluable comments on this manuscript.

Correspondence should be addressed to Dr. Jerome M. Siegel, Neurobiology Research (151-A3), Department of Psychiatry, University of California Los Angeles School of Medicine, Veterans Affairs Greater Los Angeles Healthcare System, 16111 Plummer Street, North Hills, CA 91343. E-mail: jsiegel@ucla.edu.

DOI:10.1523/JNEUROSCI.2968-07.2007

Copyright $\odot 2007$ Society for Neuroscience ～0270-6474/07/2711999-08\$15.00/0 resembles the USWS of cetaceans (Lyamin and Mukhametov, 1998). In this state, which is also known as asymmetrical slowwave sleep (ASWS), one hemisphere displays a synchronized EEG while the other hemisphere exhibits desynchronization or synchronized SWA of a lower amplitude. Little is known about the underlying mechanisms of ASWS; however, we may begin to understand the expression of this phenomenon by studying the neurotransmitter systems thought to be involved in the generation and maintenance of sleep-wake states.

Numerous neurotransmitters have been implicated in the regulation of sleep and waking in terrestrial mammals, including acetylcholine (ACh), and various biogenic amines, amino acids, and neuropeptides (Jones, 2005). Of these, ACh, derived primarily from the basal forebrain (Semba, 2000), is thought to play an important role in regulating cortical activation [EEG desynchronization during waking and rapid eye movement (REM) sleep] and behavioral arousal (Celesia and Jasper, 1966; Jasper and Tessier, 1971; Day et al., 1991; Marrosu et al., 1995; JimenezCapdeville and Dykes, 1996).

The unique sleeping pattern of fur seals provides us with the opportunity to determine which of the many physiological and neurochemical changes seen bilaterally in terrestrial mammals are linked to the EEG-defined state, and which may be related to the behavioral quiescence, cardiorespiratory changes, and sensory input reduction that typically accompany sleep. The aim of 
the present study was to examine, simultaneously in both hemispheres, the pattern of cortical ACh release across the sleep-wake cycle in northern fur seals using in vivo microdialysis and highperformance liquid chromatography (HPLC)-electrochemical detection (ECD), in combination with polysomnography.

\section{Materials and Methods}

Animals. All procedures were approved by the University of California Los Angeles and the Veterans Affairs Greater Los Angeles Healthcare System Sepulveda Animal Research Committees and were conducted in accordance with the National Institute of Health Guide for the Care and Use of Experimental Animals. All studies were performed at the Utrish Marine Station of the Severtsov Institute of Ecology and Evolution of the Russian Academy of Sciences. Data were collected from four male juvenile northern fur seals (Callorhinus ursinus; $20-25 \mathrm{~kg}, 2-3 \mathrm{yrs}$ ). Three days before surgery, an individual seal was selected and moved to a pentagonal enclosure (1.5 m per side) situated within an empty indoor laboratory pool. During this time, the seal was placed into the microdialysis recording cage $(1.1 \times 0.8 \times 0.8 \mathrm{~m})$ for $4-6 \mathrm{~h}$ each day. During the daytime (8:00 A.M. to 8:00 P.M.), the enclosure was illuminated by artificial light (400 lux); at night the level of illumination was reduced ( 80 lux). Seals were fed fish twice per day (8:00 to 9:00 A.M. and 6:00 to 7:00 P.M.). Room temperature varied between 14 and $17^{\circ} \mathrm{C}$.

Surgical procedures. One milliliter of $0.1 \%$ atropine sulfate solution was administered (i.m.) 15 min before surgery. After the initial masking ( $3 \%$ isoflurane for induction), the seal was intubated and deep anesthesia was maintained with isoflurane (0.5-2\%). Rectal temperature, heart rate, respiration rate, and oxygen saturation were continuously monitored throughout the surgery. During the initial masking, antibiotics (ciprofloxacin, $5 \mathrm{mg} / \mathrm{kg}$, i.m.) and analgesics (carprofen, $2 \mathrm{mg} / \mathrm{kg}$, s.c.) were administered.

Aseptic surgical techniques were used to implant three pairs of stainless-steel screws ( 1 x $4 \mathrm{~mm}$ ) epidurally over symmetrical occipital [anteroposterior (AP) $-20 \mathrm{~mm}$ and lateral medial (LM) $\pm 10 \mathrm{~mm}$ relative to bregma], parietal (AP $0 \mathrm{~mm}$ and $\mathrm{LM} \pm 20 \mathrm{~mm}$ relative to bregma) and frontal ( $\mathrm{AP}+20 \mathrm{~mm}$ and $\mathrm{LM} \pm 10 \mathrm{~mm}$ relative to bregma) cortical areas. These screws served as EEG electrodes. One additional screw, implanted along the midline above the nasal cavity, served as the indifferent electrode. Four Teflon-coated multistranded stainless steel wires $(0.3$ $\mathrm{mm} \times 5 \mathrm{~cm}$ ) were implanted into the nuchal muscles for electromyogram (EMG) recording. A pair of nickel-chromium insulated wires $(0.5$ $\mathrm{mm} \times 2 \mathrm{~cm}$ ) was inserted into each orbit to record electrooculogram (EOG). All leads were connected to a 19-pin socket and fixed to the skull with dental cement.

One (seal 1, seal 3), two (seal 2), or three pairs (seal 4) of stainless-steel guide cannulas $(0.5 \mathrm{~mm} \times 12$ or $18 \mathrm{~mm}$; AG-12 or AG-18; Eicom, Kyoto, Japan) were implanted in symmetrical locations in each hemisphere and secured to the skull using dental cement (Fig. 1a). An obturator (AD-12 or AD-18; Eicom) was inserted into each guide cannula and secured with a cap nut (AC-1; Eicom) until it was replaced with a microdialysis probe.

After surgery, the seal was returned to the indoor enclosure and allowed 1 week to recover before insertion of the microdialysis probes. During the recovery period, seals were given antibiotics (ciprofloxacin, 5 $\mathrm{mg} / \mathrm{kg}$ ) and analgesics (carprofen, $2 \mathrm{mg} / \mathrm{kg}$ ) with fish twice per day. Two days after the surgery, the seal was connected to the polygraph via a low noise cable. On the morning of the day before the microdialysis experiment, the seal was placed in the recording cage and remained there until insertion of the probes the next day.

Microdialysis procedure. On the morning of the experiment, the seal was anesthetized with isoflurane as described above, and the obturators were replaced with microdialysis probes. Probes had a 12 or $18 \mathrm{~mm}$ long shaft with a $2 \mathrm{~mm}$ long semipermeable artificial cellulose membrane (50 $\mathrm{kDa}$ cutoff, $0.22 \mathrm{~mm}$ outer diameter; AI-12-02 or AI-18-02; Eicom). Shielded Teflon tubing (1.5 m; TJT-150 HS; Eicom) encased within an additional protective sleeve was connected to the inlet and outlet of each probe. After the implantation procedure, the seal was placed back in the recording chamber where it was free to move around. Subsequently, the tubing connected to the probe inlet was connected to a syringe pump
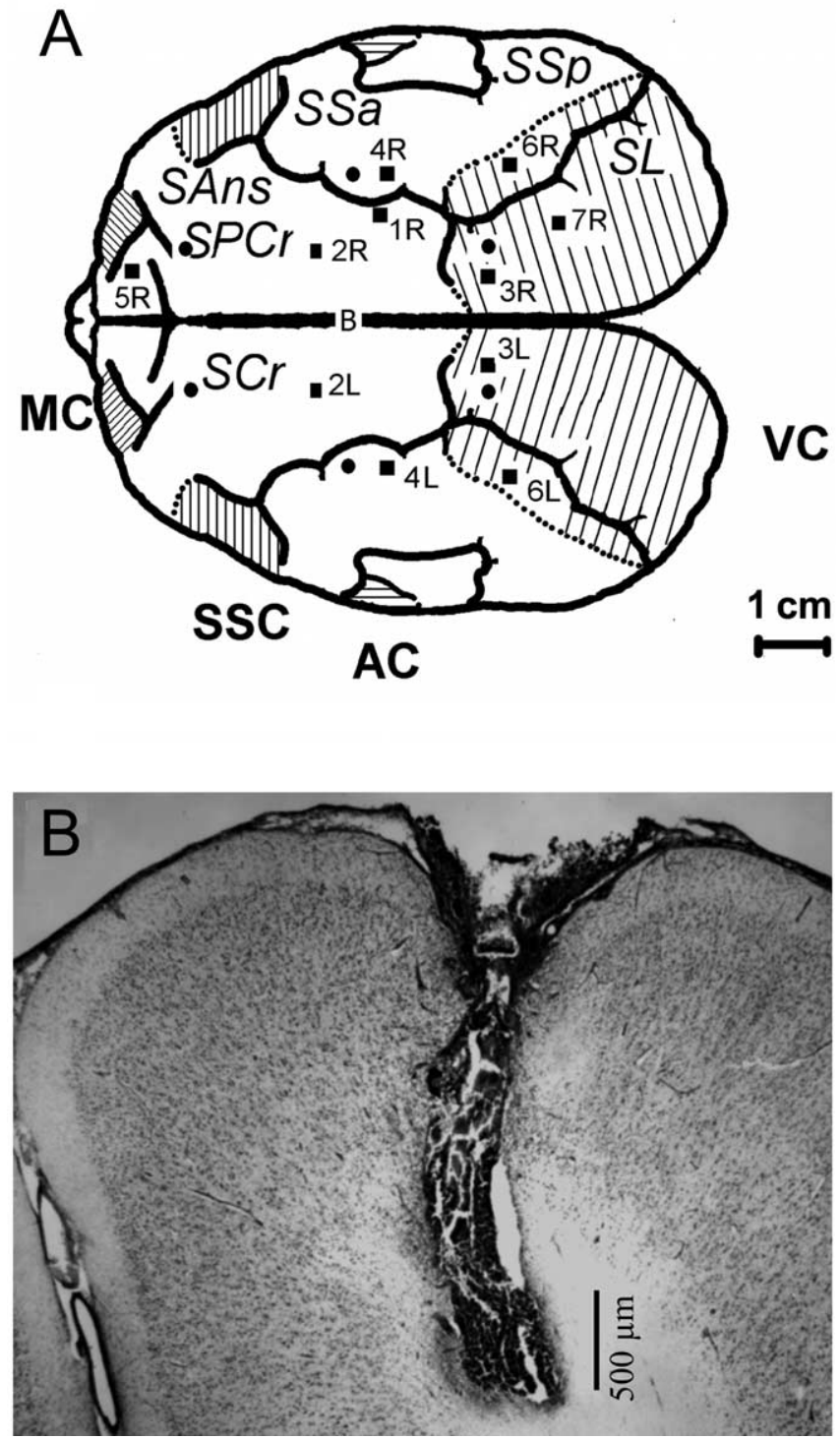

Figure 1. A, Reconstructed schematic illustrating the localization of microdialysis probes (ם) relative to the first-order sulci and EEG electrodes $(\mathbf{O}$ ) relative to bregma (B). SL, Sulcus lateralis; SSa, suprasylvian anterior; SSp, suprasylvian posterior; SANs, supraansatus; SPCs, supraprecursiatus. Positions of the main areas (line shaded) in the cerebral cortex were taken from Supin et al. (2001). VC, Visual cortex; AC, auditory cortex; SSC, sensory-motor cortex; MC, motor cortex. $\boldsymbol{B}$, Photomicrograph of a representative cresyl violet stained coronal section $(40 \mu \mathrm{m})$ depicting placement of the dialysis site within the associative cortex of a northern fur seal (probe 2L of seal 2).

(ESP-64, Eicom) and the probes were perfused with Ringer's solution $\left(147 \mathrm{mM} \mathrm{NaCl}^{2} 2.3 \mathrm{mM} \mathrm{CaCl}_{2}\right.$, and $\left.4 \mathrm{~mm} \mathrm{KCl}\right)$ containing $10 \mu \mathrm{M}$ eserine at a rate of $2 \mu \mathrm{l} / \mathrm{min}$. The tubing from the probe outlet was connected to a fraction collector (EFC-82; Eicom). During collection, samples were kept at $4^{\circ} \mathrm{C}$ (EFR-82 Cooling Unit; Eicom). Isopropylhomocholine (IPHC; Eicom) was added to each sample as an internal standard, immediately after which samples were stored at -20 or $-80^{\circ} \mathrm{C}$ until analyzed. After a $4 \mathrm{~h}$ stabilization period, samples ( 10 or $20 \mu \mathrm{l}$ ) were collected every 5 or 10 min over the course of $8-40 \mathrm{~h}$.

Assay of acetylcholine. Samples were assayed for ACh using a combination of HPLC, enzymatic reaction, and ECD (HTEC-500; Eicom). Samples, kept at $4^{\circ} \mathrm{C}$, were injected into the HPLC system using an autosampler (231 XL Sample Injector; 402 Syringe Pump; 832 Temperature Regulator; Gilson., Middleton, WI). The mobile phase consisting of 50 mm potassium bicarbonate buffer, $\mathrm{pH} 8.2$, containing $5 \mathrm{mg} / \mathrm{L}$ EDTA-2Na (Dojindo Laboratories, Kumamoto, Japan) and $400 \mathrm{mg} / \mathrm{L}$ 
1-decanesulfonic acid (Tokyo Kasei Kogyo, Tokyo, Japan) was filtered through a guard (pre) column (PC-03 with $\mathrm{CH}$-GEL packing material; Eicom) and delivered at a rate of $150 \mu \mathrm{l} / \mathrm{min}$. ACh was separated on a styrene polymer reversed-phase column [AC-GEL, $2 \mathrm{~mm}$ inner diameter (ID) $\times 150 \mathrm{~mm}$, Eicom] and subsequently catalyzed in a postcolumn enzyme reactor containing immobilized acetylcholinesterase (AChE) and choline oxidase $(\mathrm{ChO})$ (AC-enzympak, $3 \mathrm{~mm} \mathrm{ID} \times 4 \mathrm{~mm}$, Eicom) maintained at $33^{\circ} \mathrm{C}$. ACh was hydrolyzed by AChE to acetate and choline, and choline was oxidized by $\mathrm{ChO}$ to produce hydrogen peroxide and betaine. The amount of hydrogen peroxide corresponding to ACh was electrochemically detected by a platinum working electrode (WE-PT; Eicom) set to $+450 \mathrm{mV}$ against a $\mathrm{Ag}^{+} / \mathrm{AgCl}$ reference electrode (RE-500; Eicom). The signal from the detector was recorded using a data acquisition system and analyzed using PowerChrom software (EPC-300; Eicom). Before each experiment, the HPLC system was calibrated and tested for linearity using six concentrations of ACh and IPHC ranging from $10 \mathrm{fmol}$ to $1 \mathrm{pmol}$. The detection limit for ACh was $10 \mathrm{fmol} / 20 \mu \mathrm{l}$ injection with a 3:1 signal-to-noise ratio. Several times per day the HPLC system was calibrated using an external standard containing a known concentration of ACh and IPHC. Quantification of ACh in the dialysates was performed by comparison with the internal standard (IPHC) peak area.

Electroencephalogram recording and analysis. EEG from two symmetrical pairs of cortical electrodes, and EMG and EOG of one or both eyes were continuously recorded throughout the experiment. The seal was also continuously observed and videotaped by an experimenter located in an adjoining room. Bipolar EEG recordings from each hemisphere were bandpass filtered $(0.3-30.0 \mathrm{~Hz})$, amplified, digitally sampled at 200 $\mathrm{Hz}$, and stored using CED1401 Plus and Spike 2 software (Cambridge Electronic Design, Cambridge, UK). Data were scored as in our previous studies (Mukhametov et al., 1985; Lyamin and Chetyrbok, 1992; Lyamin et al., 2002b). For each hemisphere, EEG was visually scored in $20 \mathrm{~s}$ epochs as (1) desynchronization (low-amplitude, fast activity; stage 1 EEG), (2) low-amplitude synchronization [sleep spindles and slow waves $(0.5-4.0 \mathrm{~Hz})$, which exceeded stage 1 amplitude by at least 1.5 times and with waves of maximal amplitude occupying $<50 \%$ of the epoch; stage 2 EEG], or (3) high amplitude synchronization (slow waves of maximal amplitude occupying $>50 \%$ of the epoch; stage 3 EEG).

Waking was characterized by stage 1 EEG in both hemispheres combined with sustained muscle tone and/or bursts of muscle activity. AW was scored when the animal was moving around in the recording cage, grooming, or feeding. QW was scored when the seal was laying or sitting either motionless or with occasional shifts in body position during which time their eyes could be open or closed. REM sleep was scored when a desynchronized EEG typical of waking (i.e., stage 1 EEG) in both hemispheres was accompanied by a significant reduction in EMG tone and occasionally full atonia, muscle jerks, facial twitches, and rapid eye movements. BSWS was scored when stage 2 and/or stage 3 EEG appeared simultaneously in both hemispheres along with lower or similar amplitude EMG activity as seen during QW. Left asymmetrical slow-wave sleep (LASWS) was scored when stage 3 EEG occurred in the left hemisphere and stage 2 or stage 1 EEG occurred in the right hemisphere, or when stage 2 EEG occurred in the left hemisphere and stage 1 EEG occurred in the right hemisphere. Right asymmetrical slow wave sleep (RASWS) was scored when a higher level of EEG synchronization was recorded in the right hemisphere (Fig. 2). The percentage of time spent in each behavioral state was calculated for each sample. Dialysates were classified as AW, QW, or REM sleep samples when a single behavioral state occupied $75 \%$ or more of the sampling interval. Samples were classified as BSWS when SWS occurred simultaneously in both hemispheres and occupied $75 \%$ or more of the sampling interval. When SWS occupied $75 \%$ or more of the sampling interval but $<25 \%$ of it was BSWS, the sample was classified as either LASWS or RASWS. All remaining samples were classified as mixed state.

For each hemisphere, EEG spectral power in the $\delta$ frequency range (1.2-4 Hz; also known as SWA) was computed in consecutive $5 \mathrm{~s}$ epochs by fast Fourier transformation using Spike 2 software. Epochs containing artifacts were excluded from additional spectral analysis. The average EEG power $(1.2-4 \mathrm{~Hz})$ in each hemisphere was then calculated for each sample.
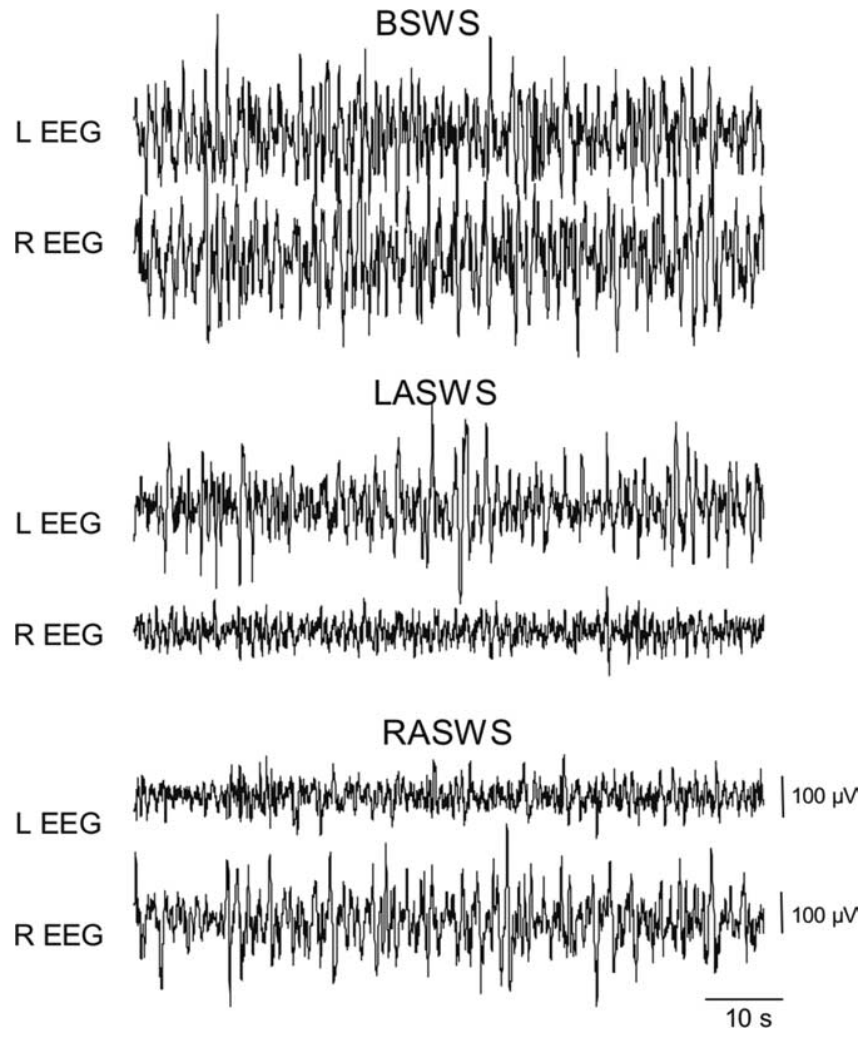

Figure 2. Cortical EEG (bipolar fronto-occipital recordings) of the left (L) and right (R) hemispheres during SWS in a northern fur seal (seal 2). Representative truncated episodes ( $60 \mathrm{~s}$ ) of BSWS and SWS with interhemispheric EEG asymmetry (LASWS and RASWS, respectively) are shown.

Histological analysis. After the completion of the experiments, animals were deeply anesthetized and perfused transcardially with $0.9 \%$ saline followed by $4 \%$ paraformaldehyde in $0.1 \mathrm{~m}$ phosphate buffer (PB), $\mathrm{pH}$ 7.4. Brains were removed and postfixed in $2 \%$ paraformaldehyde in $0.1 \mathrm{M}$ $\mathrm{PB}$. Before sectioning, brains were transferred to $25 \%$ sucrose in $0.1 \mathrm{M} \mathrm{PB}$ until equilibrated. Brain regions containing the track of the microdialysis probe were cut into $40 \mu \mathrm{m}$ coronal sections on a freezing microtome or cryostat. Sections containing the track of the microdialysis probe were mounted on slides, stained with cresyl violet, and examined microscopically to verify placement of the microdialysis probe within the cortex.

Statistical analysis. All statistical analyses were performed using SPSS 14.0 (SPSS, Chicago, IL). Results were assessed for statistical significance using either one-way ANOVA with repeated-measures followed by a Bonferroni's post hoc multiple comparisons tests, Student's $t$ test, paired $t$ test, or Pearson's correlation coefficient. The significance level was $p<$ 0.05 . Values are given as mean \pm SEM.

\section{Results}

Samples were collected from 11 microdialysis sites in four northern fur seals. Of all samples collected, 1335 were assayed for ACh content. Among these, 679 (51\%) met the criteria described above, consisting of 140 BSWS, 38 REM sleep, 199 QW, 225 AW, 48 LASWS, and 26 RASWS samples. Histological analysis verified placement of microdialysis probes within the cortex (Fig. 1b).

\section{Cortical ACh release varies across bilaterally symmetrical EEG states (BSWS, REM sleep, QW, and AW)}

Representative chromatograms of ACh peaks obtained from samples collected across the sleep-wake cycle are depicted in Figure 3a. Cortical ACh levels and the corresponding behavioral state composition for sequential samples collected over a $1 \mathrm{~h}$ period are displayed in Figure $3 b$. Cortical ACh release fluctuated 

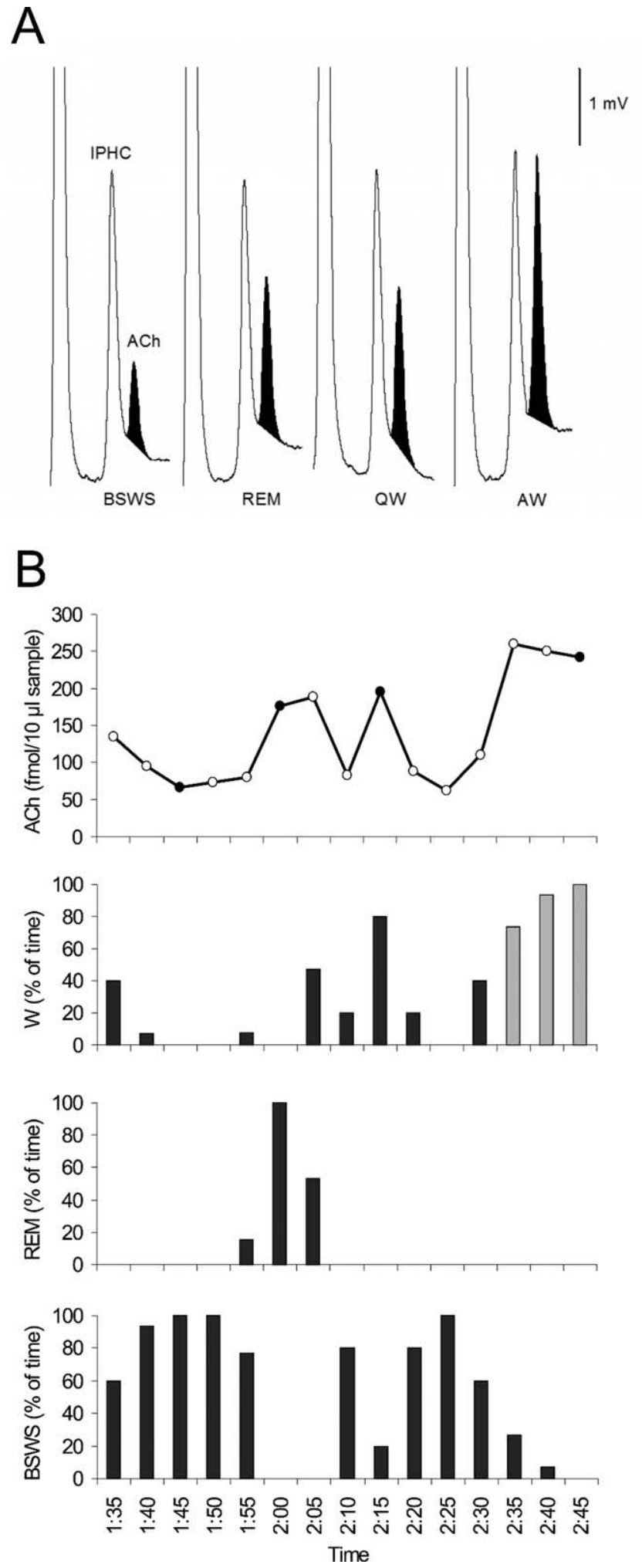

Figure 3. Cortical ACh release varied across the sleep-wake cycle in northern fur seals. $\boldsymbol{A}$, Typical chromatograms displaying the level of ACh in dialysates collected during each behavioral state (BSWS, REM, QW, and AW). ACh (darkened) eluted just after the internal standard $(\mathrm{IPHC})$ at a retention time of $13.75 \mathrm{~min}$. $\boldsymbol{B}$, Representative time course of cortical ACh levels across the sleep-wake cycle. In the ACh panel, darkened circles represent values generated from the corresponding chromatograms displayed above. In the wake (W) panel, black bars represent $\mathrm{QW}$ and gray bars represent AW. Data shown here were obtained from probe $5 \mathrm{R}$ located just posterior to the motor cortex of seal 4. substantially during this timeframe, with minimum levels corresponding to BSWS and maximal levels corresponding to AW. When all samples, for each animal, were categorized according to amount of waking and REM sleep combined during the sampling period $(<25,25-50,50-75$, or $>75 \%)$, the mean level of ACh was positively correlated with the percentage of EEG desynchronization $\left(F_{3,30}=10.33 ; p<0.0001\right)$.

ACh levels across the sleep-wake cycle for all 11 dialysis sites are summarized in Table 1. Samples were obtained during all behavioral states for 7 of the 11 probes. Mean cortical ACh release was state-dependent $\left(F_{3,18}=13.89 ; p<0.0001\right)$; cortical ACh levels were minimal during BSWS at $157 \pm 35.8 \mathrm{fmol} / 20 \mu \mathrm{l} \mathrm{sam}-$ ple $(n=95)$, increased to $346 \pm 85.8 \mathrm{fmol} / 20 \mu \mathrm{l} \mathrm{sample}(n=38)$ during REM sleep and to $335 \pm 82.7 \mathrm{fmol} / 20 \mu \mathrm{l} \mathrm{sample}(n=150)$ during QW, and were maximal during AW at $442 \pm 109 \mathrm{fmol} / 20$ $\mu$ sample $(n=180)$. When compared with BSWS, ACh levels increased by $216 \%$ during REM sleep, $210 \%$ during QW, and $283 \%$ during AW (Fig. 4). Post hoc tests revealed that ACh release was: significantly higher during REM sleep, $\mathrm{QW}$, and $\mathrm{AW}$ when compared with BSWS (all $p$ values $<0.0001$ ), not significantly different during REM sleep and QW ( $p=0.536)$, and significantly elevated during AW when compared with REM sleep ( $p=$ $0.020)$ and QW ( $p=0.007)$ levels. For the four probes in which no REM sleep samples were obtained (Table 1), cortical ACh release followed a similar trend.

For each pair of symmetrically placed probes, changes in ACh release occurred synchronously in both hemispheres during bilaterally symmetrical states (all $r$ values were $\geq 0.810$ and all $p$ values were $<0.0001$ ) (Fig. $5 a$ ). For each seal, changes in ACh release from probes placed within different cortical areas within the same hemisphere paralleled one another regardless of behavioral state (all $r$ values were $\geq 0.722$ and all $p$ values were $<0.0001$ ) (Fig. 5b).

When probes were grouped into two categories according to cortical location (Fig. 1a, Table 1), mean ACh release for each behavioral state was not significantly different between probes located in the associative and visual cortex (all $p$ values were $\geq 0.172$ ). ACh levels obtained from the single probe located $<5$ $\mathrm{mm}$ posterior to the motor cortex were elevated compared with mean values from the association and visual cortex.

\section{Cortical ACh release is lateralized during asymmetrical EEG states (LASWS and RASWS)}

Three fur seals displayed ASWS during the observation period (seals 1,2 , and 4 ). For all animals, EEG power in the $\delta$ frequency range $(1.2-4 \mathrm{~Hz})$ was maximally expressed in both hemispheres during BSWS and minimally expressed at similar levels during QW and REM sleep. During LASWS, EEG power $(1.2-4 \mathrm{~Hz})$ was higher in the left hemisphere compared with the right hemisphere (Fig. 6a). During this state, EEG power $(1.2-4 \mathrm{~Hz})$ in the left hemisphere approached, but generally did not reach, the level observed during BSWS whereas EEG power $(1.2-4 \mathrm{~Hz})$ in the right hemisphere approached, but was typically greater than the level observed for QW (Fig. 6a). Similar trends, albeit in opposite hemispheres, were observed during RASWS (Fig. 6a). For all animals, ACh release was lateralized during ASWS with greater release in the hemisphere displaying lower voltage activity [i.e., lower EEG power (1.2-4 Hz)] (Fig. 6b). EEG power (1.2-4 Hz) and cortical ACh release in the same hemisphere were negatively correlated (Fig. 7). However, there was no correlation between EEG power $(1.2-4 \mathrm{~Hz})$ and cortical ACh release in the contralateral hemisphere (Fig. 7). 
Table 1. Mean \pm SEM cortical ACh release during BSWS, REM sleep, QW, and AW and the percentage changes in ACh levels from BSWS for 11 microdialysis probes in four northern fur seals

\begin{tabular}{|c|c|c|c|c|c|c|c|c|c|}
\hline \multirow[b]{2}{*}{ Seal } & \multirow[b]{2}{*}{ Probe $^{a}$} & \multirow[b]{2}{*}{ Location $^{b}$} & \multicolumn{4}{|c|}{ ACh (fmol/20 $\mu$ l sample) } & \multicolumn{3}{|c|}{ ACh (\% of BSWS) } \\
\hline & & & BSWS & REM & QW & AW & REM & QW & AW \\
\hline 1 & $1 R^{c}$ & AsC & $55 \pm 6(10)$ & $96 \pm 23(3)$ & $87 \pm 3(11)$ & $144 \pm 8(5)$ & 175 & 158 & 262 \\
\hline 2 & $2 \mathrm{~L}^{d}$ & AsC & $139 \pm 14(12)$ & & $351 \pm 26(13)$ & $450 \pm 16(10)$ & & 253 & 324 \\
\hline 2 & $2 R^{d}$ & AsC & $22 \pm 3(10)$ & & $57 \pm 9(10)$ & $78 \pm 9(9)$ & & 259 & 355 \\
\hline 2 & $3 \mathrm{~L}^{d}$ & VC & $88 \pm 11(12)$ & & $250 \pm 21(13)$ & $388 \pm 10(10)$ & & 284 & 441 \\
\hline 2 & $3 \mathrm{R}^{d}$ & VC & $56 \pm 7(11)$ & & $149 \pm 14(13)$ & $180 \pm 3(10)$ & & 266 & 321 \\
\hline 3 & $4 \mathrm{~L}$ & AsC & $98 \pm 12(7)$ & $218 \pm 38(3)$ & $224 \pm 25(10)$ & $328 \pm 33(7)$ & 222 & 229 & 335 \\
\hline 3 & $4 R$ & AsC & $127 \pm 13(7)$ & $259 \pm 26(3)$ & $258 \pm 20(11)$ & $340 \pm 31(7)$ & 204 & 203 & 268 \\
\hline $4^{d}$ & $5 \mathrm{R}^{c, f}$ & $\mathrm{MC}^{g}$ & $176 \pm 6(25)$ & $410 \pm 25(11)$ & $406 \pm 5(93)$ & $566 \pm 7(137)$ & 233 & 231 & 322 \\
\hline $4^{e}$ & $6 \mathrm{~L}$ & VC & $167 \pm 18(16)$ & $387 \pm 25(6)$ & $362 \pm 91(9)$ & $379 \pm 89(9)$ & 230 & 215 & 226 \\
\hline $4^{e}$ & $6 \mathrm{R}$ & VC & $118 \pm 17(14)$ & $248 \pm 50(6)$ & $237 \pm 52(8)$ & $304 \pm 60(11)$ & 210 & 201 & 258 \\
\hline $4^{e}$ & $7 R^{c}$ & VC & $334 \pm 37(16)$ & $802 \pm 65(6)$ & $774 \pm 121(8)$ & $1035 \pm 145(10)$ & 240 & 232 & 310 \\
\hline
\end{tabular}

The number of samples is shown in parentheses. AsC, Associative cortex; $V C$, visual cortex; $M C$, motor cortex; L, left; $R$, right.

${ }^{a}$ Probe number and hemisphere (Hem). Each seal was given a unique but consecutive probe number. Within a given seal, probes with the same number were placed in symmetrical locations within each hemisphere. When more than one probe was implanted in a single hemisphere, the probe number is unique but the hemisphere is the same.

${ }^{b}$ Probe location (see Fig. 1a).

'No data was obtained for the corresponding probe in the left hemisphere because of technical difficulties.

${ }^{d, e}$ Two separate experiments were conducted on this seal.

fSamples were collected in 5 min increments for a volume of $10 \mu \mathrm{l}$. As a result, concentrations were scaled up to $20 \mu \mathrm{l}$.

${ }^{9}$ Located slightly posterior to the motor cortex (see Fig. 1a).

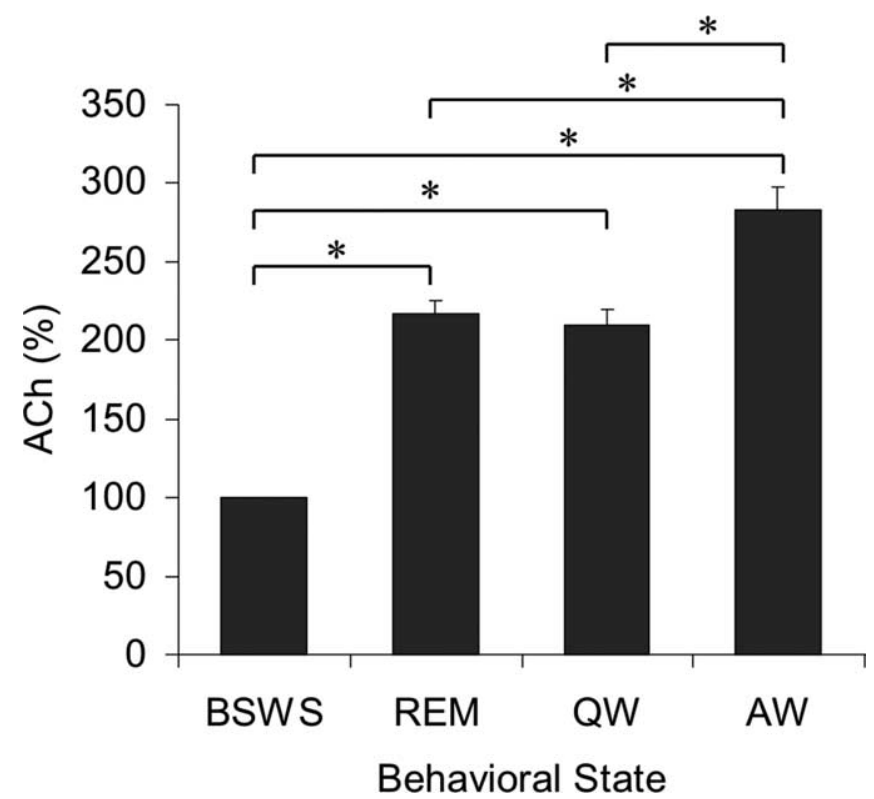

Figure 4. Cortical ACh release differed significantly across the sleep-wake states (BSWS, REM, QW, and AW) in northern fur seals. ACh levels during REM sleep and QW were not significantly different $(p=0.536)$. However, all other comparisons were statistically significant $\left({ }^{*} p<0.05\right)$. Each column represents the overall mean ( \pm SEM) of percentage changes in ACh levels relative to BSWS. Data shown here were obtained from seven dialysis sites in three seals (Table 1).

\section{Discussion}

In the present study we determined that cortical ACh release (1) varied across the sleep-wake cycle, and (2) was lateralized during ASWS in the northern fur seal. This is the first in vivo neurochemical investigation in a marine mammal. Furthermore, this is the first study, to our knowledge, showing that neurochemical asymmetry exists in an animal under natural physiological conditions.

Previously, cortical ACh release had been measured only in terrestrial mammals which display bilaterally symmetrical EEG states (Jasper and Tessier, 1971; Marrosu et al., 1995). During such states in the fur seal, cortical ACh release exhibited the following pattern: AW $>$ QW $=$ REM $>$ BSWS. The same state- dependent pattern has been observed previously in the bilateral sleep of cats (Jasper and Tessier, 1971; Marrosu et al., 1995). Furthermore, changes in the magnitude of ACh release found in this study closely followed those obtained in the aforementioned studies when calculated relative to SWS. Using methods similar to those used in this study, Marrosu et al. (1995) found that cortical ACh levels were maximal during AW at 280\% relative to SWS, and increased to a similar level during QW and REM sleep at 229 and $216 \%$, respectively. Our results are also consistent with data obtained using the original ACh bioassay, the cortical cup technique, which showed that ACh release was similarly elevated during waking and REM sleep, increasing by 175 and 183\%, respectively, when compared with SWS (Jasper and Tessier, 1971). This previous investigation did not subdivide wakefulness into AW or QW. Together, these studies suggest that changes in cortical ACh release during bilaterally symmetrical EEG states are conserved between terrestrial and marine mammals.

A previous study determined that cholinergic basal forebrain neurons were active during both waking and REM sleep, and virtually silent during SWS in rats (Lee et al. 2005), with an average discharge rate pattern of: $\mathrm{REM}>\mathrm{AW}>\mathrm{QW}>\mathrm{SWS}$. This would suggest that cortical ACh release would be maximal during REM sleep. However this was not the case for this study, nor that of Marrosu et al. (1995), suggesting a nonlinear relationship between overall discharge rate of cholinergic projection neurons and $\mathrm{ACh}$ release in the cortex.

Unlike fully terrestrial or aquatic mammals, northern fur seals display both bilateral and asymmetrical EEG states, providing a single, intact system in which to study the neurochemical correlates of the EEG changes across the sleep-wake cycle. In studying this unique feature of the fur seal, we can dissociate the thalamocortical EEG from aspects of behavioral state and study each independently as they relate to neurotransmitter release. Our data show that during ASWS, cortical ACh release is lateralized, with maximal release in the hemisphere displaying lower-voltage activity. This study provides evidence that the reduction in cortical ACh release is inversely linked to hemispheric EEG amplitude, but is not a reflection of the behavioral quiescence, sensory input reduction, or cardiorespiratory changes that accompany sleep.

We do not know the neuroanatomical substrates of ASWS, 

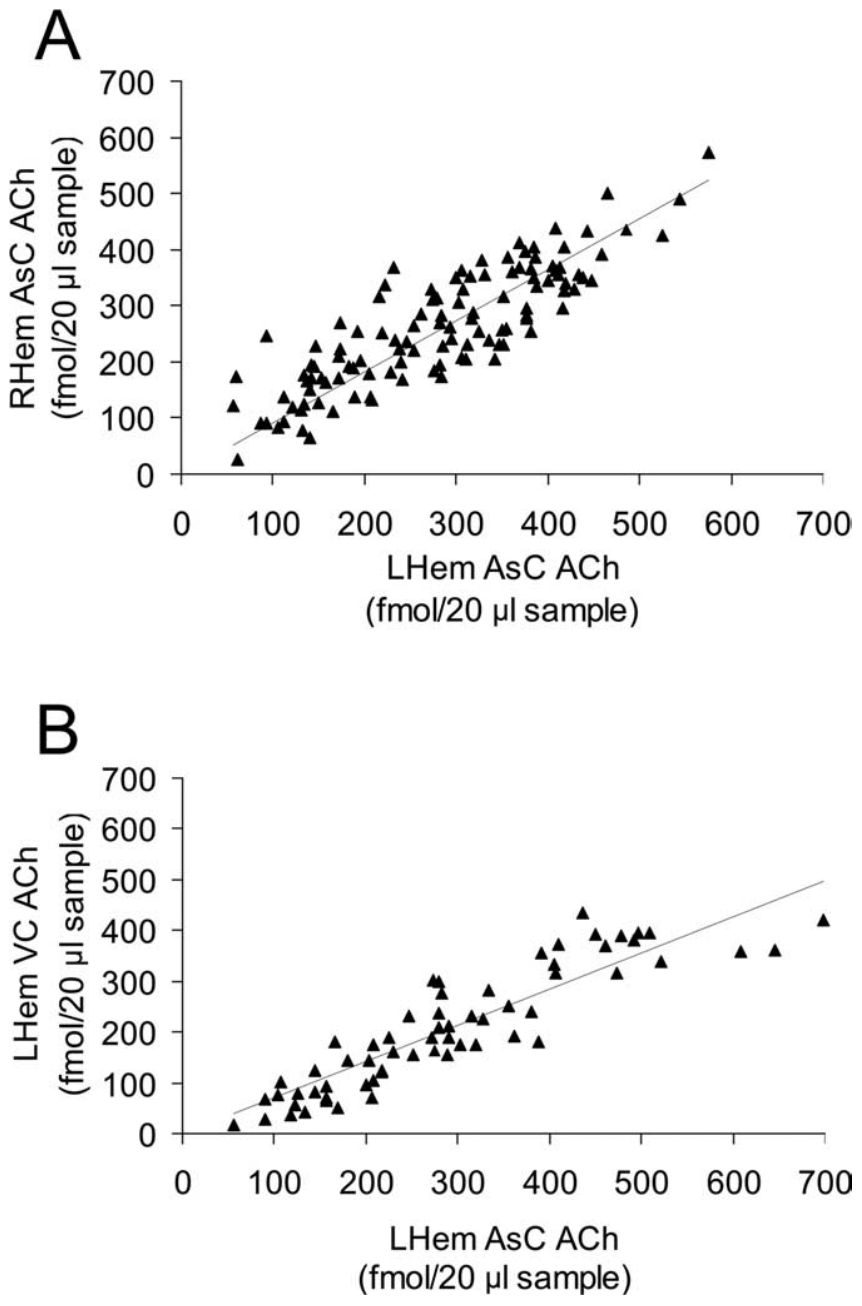

Figure 5. $A$, Changes in cortical ACh release were strongly and positively correlated between hemispheres during bilaterally symmetrical behavioral states (i.e., AW, QW, BSWS, REM) $\left(r_{116}\right.$ $=0.855 ; p<0.0001)$. LHem, Left hemisphere; RHem, right hemisphere. Data shown here were obtained from probes $4 \mathrm{~L}$ and $4 \mathrm{R}$ located within the associative cortex (AsC) of seal 3. B, Regardless of behavioral state, changes in cortical ACh release were strongly and positively correlated within a single hemisphere $\left(r_{61}=0.850 ; p<0.0001\right)$. Data shown here were obtained from probes $2 \mathrm{~L}$ and $3 \mathrm{~L}$ located within the AsC and visual cortex (VC), respectively, of seal 2.

nor do we know the structures responsible for lateralized ACh release during this state. The parallel asymmetries of the EEG and cortical ACh release, however, point to a role for subcortical structures in the generation of both of these conditions. Across mammals, the nucleus basalis of the basal forebrain is considered the primary source of ACh released in the cortex (Semba, 2004) and this projection is ipsilateral (Bigl et al. 1982). Intrinsic cortical cholinergic neurons are present in some species, but not in others, and are presumed to be a minor source of ACh (Semba, 2004). In rats, unilateral electrical stimulation of the nucleus basalis is associated with an ipsilateral increase in cortical ACh release (Casamenti et al., 1986; Kurosawa et al., 1989; Rasmusson et al., 1992; Jimenez-Capdeville et al., 1997) and EEG activation (Casamenti et al., 1986; Buzsaki et al., 1988; Metherate et al., 1992; Kleiner and Bringmann, 1996) although the contralateral cortex remains unchanged. Conversely, unilateral lesions in this area result in an ipsilateral reduction in cortical ACh release (Lo Conte et al., 1982; Herrera-Marschitz et al., 1990; Dekker and Thal, 1993), concomitant with greater EEG synchronization (Lo
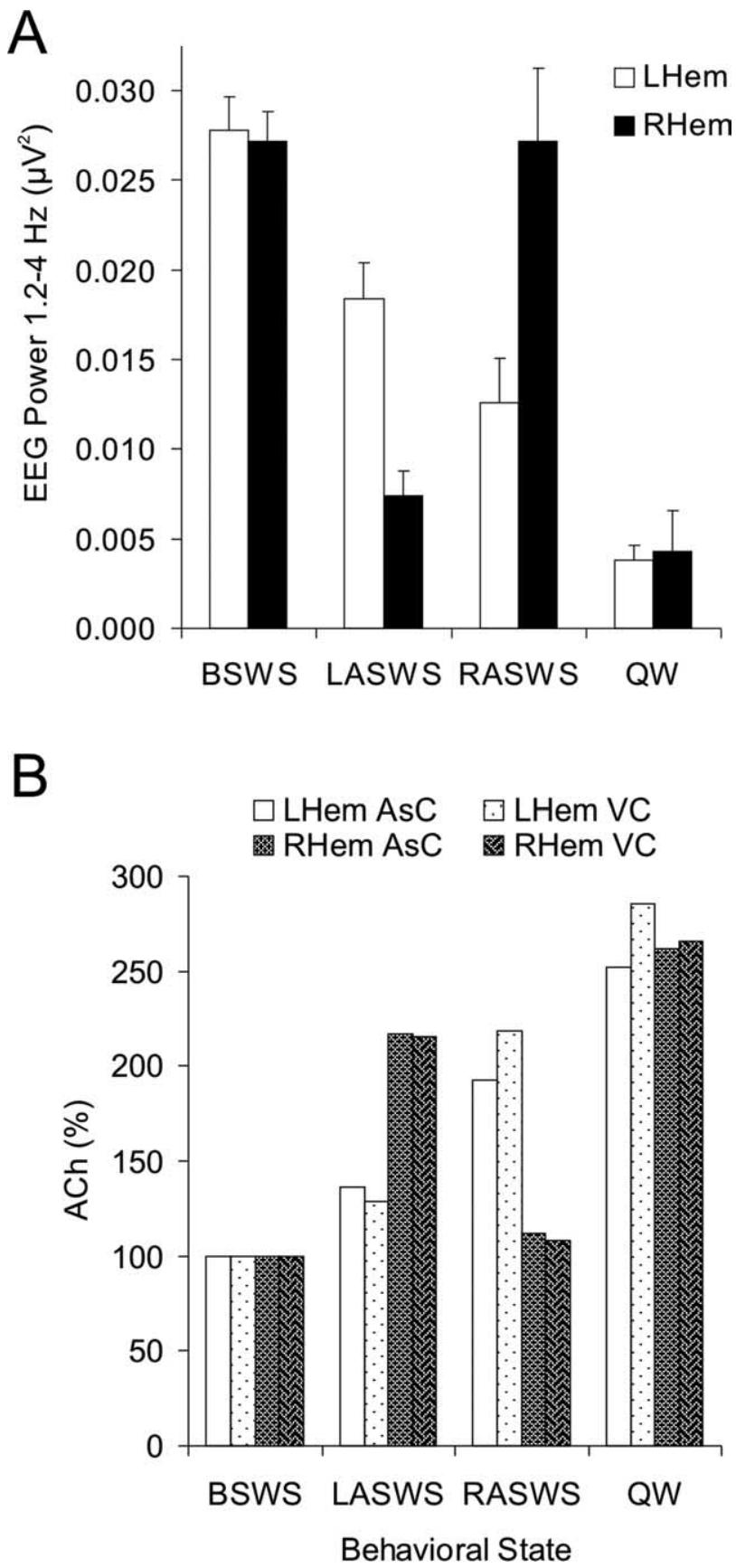

Figure 6. A, During LASWS, EEG power in the $1.2-4 \mathrm{~Hz}$ frequency range was significantly higher in the left hemisphere (LHem) compared with that observed in the right hemisphere $\left(\right.$ RHem; $\left.t_{(12)}=4.447 ; p<0.0002\right)$. The opposite was observed during RASWS with EEG power $(1.2-4 \mathrm{~Hz})$ being significantly higher in the right hemisphere $\left(t_{(7)}=-3.119 ; p<0.0089\right)$. $\boldsymbol{B}$, During LASWS, cortical ACh levels in the LHem were similar to those observed during BSWS $\left(t_{(44)}\right.$ $=-1.820 ; p=0.076)$ whereas ACh levels in the RHem were comparable with those observed during $\mathrm{QW}\left(t_{(40)}=1.702 ; p=0.096\right)$. During RASWS, ACh levels in the RHem were equivalent to those observed during BSWS $\left(t_{(30)}=-0.581 ; p=0.566\right)$ whereas ACh levels in the LHem were slightly less than those observed during $\mathrm{QW}\left(t_{(38)}=2.677 ; p=0.011\right)$. Each column represents the mean percentage changes in ACh levels relative to BSWS. Data shown here were obtained from two probes (2L and $2 \mathrm{R}$ ) located within the associative cortex (AsC) and two probes ( $3 \mathrm{~L}$ and $3 \mathrm{R}$ ) located within the visual cortex (VC) of seal 2 . The number of dialysates was as follows: BSWS, $n=12$; LASWS, $n=11$; RASWS, $n=7 ; 0 \mathrm{QW}, n=13$.

Conte et al., 1982; Buzsaki et al., 1988; Riekkinen et al., 1990). These data indicate that ACh release contributes to EEG asymmetry between the two hemispheres. The lateralized ACh release seen in the fur seal suggests that neurons controlling the basal 

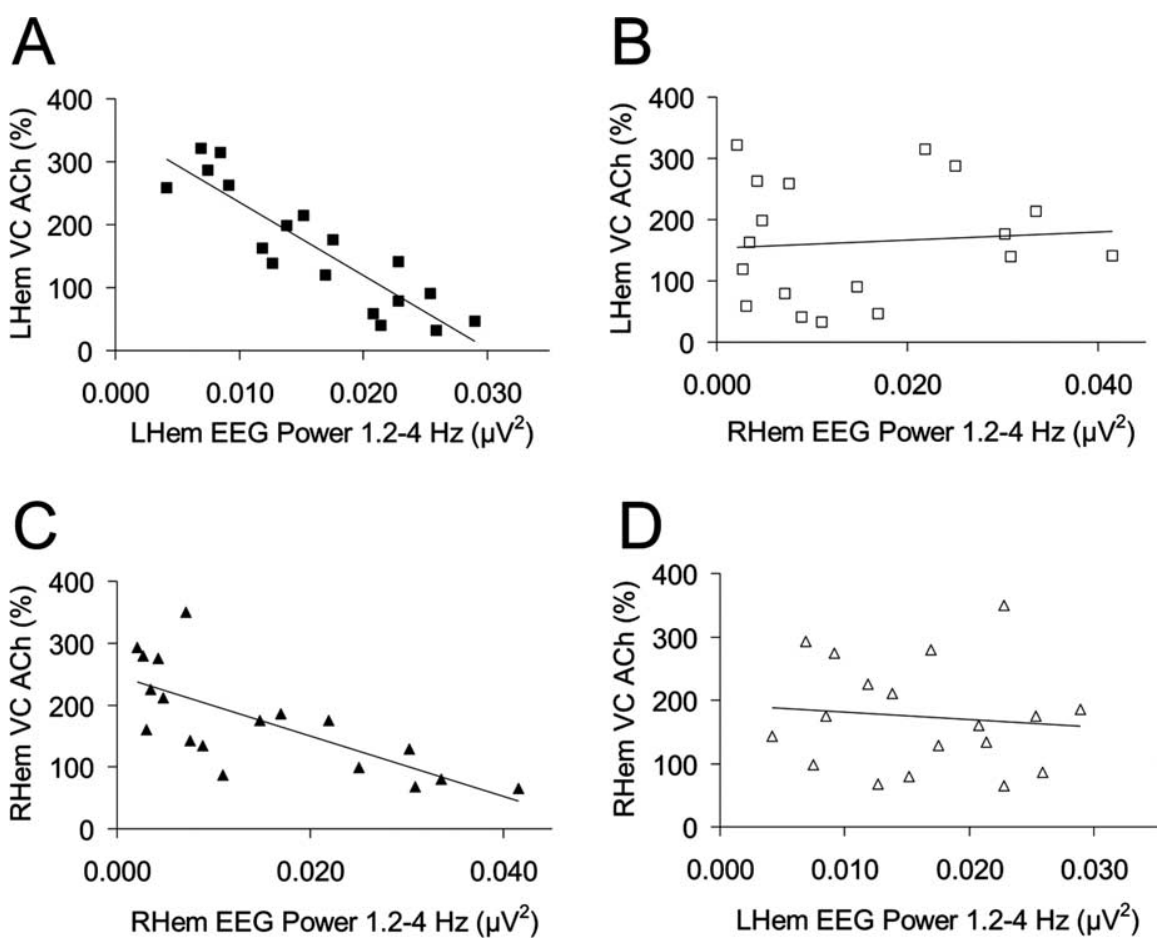

Figure 7. $\boldsymbol{A}, \boldsymbol{C}$, During ASWS, average EEG power, in the $\delta$ frequency band (1.2-4 Hz), and cortical ACh release in the same hemisphere were negatively correlated $\left(A, r_{18}=-0.896, p<0.0001 ; C, r_{18}=-0.725, p=0.001\right)$. LHem, Left hemisphere; RHem, right hemisphere. $\boldsymbol{B}, \boldsymbol{D}$, However, there was no correlation between EEG power $(1.2-4 \mathrm{~Hz})$ and cortical ACh release in the contralateral hemisphere $\left(\boldsymbol{B}, r_{18}=0.086, p=0.734 ; \boldsymbol{D}, r_{18}=-0.108, p=0.670\right)$. Data are of the visual cortex (VC) ASWS samples displayed in Figure $5 b$. Similar results were obtained for the two probes located within the associative cortex (data not shown).

forebrain ACh neuronal population act bilaterally in terrestrial mammals, but unilaterally in the fur seal and perhaps in other marine mammals with ASWS. It remains to be determined whether the norepinephrine, serotonin, and other neuronal groups that project or act bilaterally in terrestrial mammals across the sleep-wake cycle, are activated unilaterally or bilaterally in marine mammals with ASWS.

In the bottlenose dolphin, electrophysiological studies showed that SWA in the thalamus was also asymmetrical and evolved synchronously with the ipsilateral neocortex (Supin and Mukhametov, 1986). Asymmetries both at the level of the thalamus (as measured in dolphins) and the basal forebrain (as inferred by ACh release in this study) are thus consistent with a model in which the signals for ASWS/USWS originate in the brainstem. This model is further supported by research in cats, in which asynchronous SWS was induced in each hemisphere only after sagittal transection of the lower brainstem (Michel, 1972). In the current study, we also found that ACh release at different sites within the same hemisphere paralleled one another, suggesting that lateralization is not the result of a discrete, localized difference within the cortical cholinergic system.

Because sleep differs across the animal kingdom, we can benefit in our understanding of its core underlying processes and accompanying epiphenomena by exploiting such differences in a variety of animal models. The fur seal, in its expression of both ASWS and BSWS, is a valuable model from which we can dissect out the mechanisms responsible for EEG-defined sleep from those of other sleep-related behaviors. Although our study focused on the cholinergic system, a number of other neurotransmitter systems are also involved in sleep-wake states. Future work with some of these other systems in marine mammals could elucidate how sleep-related behaviors are regulated and also shed light on the question of what drives mammalian sleep.

\section{References}

Bigl V, Woolf NJ, Butcher LL (1982) Cholinergic projections from the basal forebrain to frontal, parietal, temporal, occipital, and cingulate cortices: a combined fluorescent tracer and acetylcholinesterase analysis. Brain Res Bull 8:727-749.

Buzsaki G, Bickford RG, Ponomareff G, Thal LJ, Mandel R, Gage FH (1988) Nucleus basalis and thalamic control of neocortical activity in the freely moving rat. J Neurosci 8:4007-4026.

Casamenti F, Deffenu G, Abbamondi AL, Pepeu G (1986) Changes in cortical acetylcholine output induced by modulation of the nucleus basalis. Brain Res Bull 16:689-695.

Celesia GG, Jasper HH (1966) Acetylcholine released from cerebral cortex in relation to state of activation. Neurology 16:1053-1063.

Day J, Damsma G, Fibiger HC (1991) Cholinergic activity in the rat hippocampus, cortex, and striatum correlates with locomotor activity: an in vivo microdialysis study. Pharmacol Biochem Behav 38:723-729.

Dekker AJ, Thal LJ (1993) Independent effects of cholinergic and serotonergic lesions on acetylcholine and serotonin release in the neocortex of the rat. Neurochem Res 18:277-283.

Herrera-Marschitz M, Goiny M, Utsumi H, Ferre S, Hakansson L, Nordberg A, Ungerstedt U (1990) Effect of unilateral nucleus basalis lesion on cortical and striatal acetylcholine and dopamine release monitored in vivo with microdialysis. Neurosci Lett 110:172-179.

Jasper HH, Tessier J (1971) Acetylcholine liberation from cerebral cortex during paradoxical (REM) sleep. Science 172:601-602.

Jimenez-Capdeville ME, Dykes RW (1996) Changes in cortical acetylcholine release in the rat during day and night: differences between motor and sensory areas. Neuroscience 71:567-579.

Jimenez-Capdeville ME, Dykes RW, Myasnikov AA (1997) Differential control of cortical activity by the basal forebrain in rats: a role for both cholinergic and inhibitory influences. J Comp Neurol 381:53-67.

Jones BE (2005) Basic mechanisms of sleep-wake states. In: Principles and practice of sleep medicine (Kryger MH, Roth T, Dement WC, eds), pp 136-153. Philadelphia: Elsevier Saunders.

Kleiner S, Bringmann A (1996) Nucleus basalis magnocellularis and pedunculopontine tegmental nucleus: control of the slow EEG waves in rats. Arch Ital Biol 134:153-167.

Kurosawa M, Sato A, Sato Y (1989) Stimulation of the nucleus basalis of Meynert increases acetylcholine release in the cerebral cortex in rats. Neurosci Lett 98:45-50.

Lee MG, Hassani OK, Alonso A, Jones BE (2005) Cholinergic basal forebrain neurons burst with theta during waking and paradoxical sleep. J Neurosci 25:4365-4369.

Lo Conte G, Casamenti F, Bigl V, Milaneschi E, Pepeu G (1982) Effect of magnocellular forebrain nuclei lesions on acetylcholine output from the cerebral cortex, electrocorticogram and behaviour. Arch Ital Biol 120:176-188.

Lyamin OI, Chetyrbok IS (1992) Unilateral EEG activation during sleep in the Cape fur seal, Arctocephalus pusillus. Neurosci Lett 143:263-266.

Lyamin OI, Mukhametov LM (1998) Organization of sleep in the northern fur seal. In: The northern fur seal. Systematic, morphology, ecology, behavior (Sokolov VE, Aristov AA, Lisitzjna TU, eds), pp 280-302 (in Russian). Moscow: Nauka.

Lyamin OI, Mukhametov LM, Siegel JM, Nazarenko EA, Polyakova IG, Shpak OV (2002a) Unihemispheric slow wave sleep and the state of the eyes in a white whale. Behav Brain Res 129:125-129.

Lyamin OI, Mukhametov LM, Chetyrbok IS, Vassiliev AV (2002b) Sleep and wakefulness in the southern sea lion. Behav Brain Res 128:129-138. 
Marrosu F, Portas C, Mascia MS, Casu MA, Fa M, Giagheddu M, Imperato A, Gessa GL (1995) Microdialysis measurement of cortical and hippocampal acetylcholine release during sleep-wake cycle in freely moving cats. Brain Res 671:329-332.

Metherate R, Cox CL, Ashe JH (1992) Cellular bases of neocortical activation: modulation of neural oscillations by the nucleus basalis and endogenous acetylcholine. J Neurosci 12:4701-4711.

Michel F (1972) Sleep and waking in cats with various sagittal sections of the brain. In: Cerebral interhemispheric relations (Cernacek J, Podivinsky F, eds), pp 83-97. Bratislava: Slovak Academy of Sciences.

Mukhametov LM (1984) Sleep in marine mammals. Exp Brain Res $8: 227-238$.

Mukhametov LM (1987) Unihemispheric slow-wave sleep in the Amazonian dolphin, Inia geoffrensis. Neurosci Lett 79:128-132.

Mukhametov LM, Supin AY, Polyakova IG (1977) Interhemispheric asymmetry of the electroencephalographic sleep patterns in dolphins. Brain Res 134:581-584.

Mukhametov LM, Lyamin OI, Polyakova IG (1985) Interhemispheric asynchrony of the sleep EEG in northern fur seals. Experientia 41:1034-1035.

Rasmusson DD, Clow K, Szerb JC (1992) Frequency-dependent increase in cortical acetylcholine release evoked by stimulation of the nucleus basalis magnocellularis in the rat. Brain Res 594:150-154.

Ridgway SH (2002) Asymmetry and symmetry in brain waves from dolphin left and right hemispheres: some observations after anesthesia, during quiescent hanging behavior, and during visual obstruction. Brain Behav Evol 60:265-274.

Riekkinen Jr P, Sirvio J, Miettinen R, Riekkinen P (1990) Interaction between raphe dorsalis and nucleus basalis magnocellularis in the regulation of high-voltage spindle activity in rat neocortex. Brain Res 526:31-36.

Semba K (2000) Multiple output pathways of the basal forebrain: organization, chemical heterogeneity, and roles in vigilance. Behav Brain Res 115:117-141.

Semba K (2004) Phylogenetic and ontogenetic aspects of the basal forebrain cholinergic neurons and their innervation of the cerebral cortex. Prog Brain Res 145:3-43.

Supin AY, Mukhametov LM (1986) Some mechanisms of unihemispheric slow wave sleep in dolphins. In: Electrophysiology of sensory systems in marine mammals (Sokolov VE, ed), pp 188-207 (in Russian). Moscow: Nauka.

Supin AY, Popov VV, Mass AM (2001) The sensory physiology of aquatic mammals. Boston: Kluwer Academic. 